\title{
Article \\ Evaluation of Fluorescence Intensity and Antitumor Effect Using Real-Time Imaging in Photoimmunotherapy
}

\author{
Kenji Takashima 1,2, Yoshikatsu Koga ${ }^{3} \mathbb{D}$, Takahiro Anzai ${ }^{4} \mathbb{D}$, Kayo Migita 1,5, Toru Yamaguchi 1,5, \\ Akihiro Ishikawa ${ }^{1,5}$, Shingo Sakashita ${ }^{6} \mathbb{D}$, Masahiro Yasunaga ${ }^{4}$ and Tomonori Yano ${ }^{1,2, *}$
}

1 Department of Gastroenterology and Endoscopy, National Cancer Center Hospital East, Kashiwa 277-8577, Japan; ktakashi@east.ncc.go.jp (K.T.); kmigita@east.ncc.go.jp (K.M.); toryamag@east.ncc.go.jp (T.Y.); akishika@east.ncc.go.jp (A.I.)

2 NEXT Medical Device Innovation Center, National Cancer Center Hospital East, Kashiwa 277-8577, Japan

3 Department of Strategic Programs, Exploratory Oncology Research \& Clinical Trial Center, National Cancer Center, Kashiwa 277-8577, Japan; ykoga@east.ncc.go.jp

4 Division of Developmental Therapeutics, Exploratory Oncology Research \& Clinical Trial Center, National Cancer Center, Kashiwa 277-8577, Japan; taanzai@east.ncc.go.jp (T.A.); mayasuna@east.ncc.go.jp (M.Y.)

5 Shimadzu Corporation, Kyoto 604-8511, Japan

6 Division of Developmental Pathology, Exploratory Oncology Research \& Clinical Trial Center, National Cancer Center, Kashiwa 277-8577, Japan; ssakashi@east.ncc.go.jp

* Correspondence: toyano@east.ncc.go.jp

Citation: Takashima, K.; Koga, Y.; Anzai, T.; Migita, K.; Yamaguchi, T.; Ishikawa, A.; Sakashita, S.; Yasunaga, M.; Yano, T. Evaluation of Fluorescence Intensity and Antitumor Effect Using Real-Time Imaging in Photoimmunotherapy. Pharmaceuticals 2022, 15, 223. https://doi.org/10.3390/ph15020223

Academic Editor:

Massimiliano Tognolini

Received: 14 January 2022

Accepted: 10 February 2022

Published: 14 February 2022

Publisher's Note: MDPI stays neutral with regard to jurisdictional claims in published maps and institutional affiliations.

Copyright: (C) 2022 by the authors. Licensee MDPI, Basel, Switzerland. This article is an open access article distributed under the terms and conditions of the Creative Commons Attribution (CC BY) license (https:// creativecommons.org/licenses/by/ $4.0 /)$.

\begin{abstract}
Photoimmunotherapy (PIT) is a promising tumor-selective treatment method that uses light-absorbing dye-conjugated antibodies and light irradiation. It has been reported that IR700 fluorescence changes with light irradiation. The purpose of this study was to investigate the fluorescence intensity and antitumor effect of PIT using real-time fluorescence observation of tumors and predict the required irradiation dose. The near-infrared camera system LIGHTVISION was used to image IR700 during PIT treatment. IR700 showed a sharp decrease in fluorescence intensity in the early stage of treatment and almost reached a plateau at an irradiation dose of $40 \mathrm{~J} / \mathrm{cm}$. Cetuximab-PIT for A431 xenografts was performed at multiple doses from 0-100 J/cm. A significant antitumor effect was observed at $40 \mathrm{~J} / \mathrm{cm}$ compared to no irradiation, and there was no significant difference between $40 \mathrm{~J} / \mathrm{cm}$ and $100 \mathrm{~J} / \mathrm{cm}$. These results suggest that the rate of decay of the tumor fluorescence intensity correlates with the antitumor effect by real-time fluorescence imaging during PIT. In addition, when the fluorescence intensity of the tumor plateaued in real-time fluorescence imaging, it was assumed that the laser dose was necessary for treatment.
\end{abstract}

Keywords: Photoimmunotherapy; antibody-drug conjugate; real-time imaging; laser irradiation dose

\section{Introduction}

Photoimmunotherapy (PIT) is an innovative cancer treatment method that utilizes a monoclonal antibody $(\mathrm{mAb})$ conjugated near-infrared photosensitive substances such as IR700, and light irradiation [1]. During PIT, mAb-IR700 binds specifically to antigens overexpressed in the cancer cell membrane. When exposed to $690 \mathrm{~nm}$ light, the physical properties of IR700 change, causing the membrane antigen-antibody complex to denature and aggregate. As a result, the cell membrane is damaged, causing rapid necrosis and cell death $[2,3]$. PIT has been investigated in basic experiments with various antibodies and cancer cells and has been found to be an innovative therapy that can selectively treat only the cancer cells to which the antibody binds [4-12].

In clinical practice, PIT has been most commonly used in head and neck cancer, where cancer is close to the body surface, and light irradiation is easy to perform. Moreover, phase III clinical trials are underway worldwide for patients with head and neck cancer using the EGFR-targeting cetuximab-IR700 (Cet-IR700). In addition, in September 2020, PIT was 
approved by the Pharmaceuticals and Medical Devices Agency (PMDA) in Japan to treat "unresectable, locally advanced or locally recurrent head and neck cancer" based on the results of a phase I trial ahead of other countries [13].

During PIT, it is important to administer the entire light dose only to the tumor. Therefore, there is a need to develop imaging methods that can accurately detect the extent of the tumor and determine the amount of light irradiation that will produce therapeutic effects. Fluorescence imaging is a method of observing cancer cells in real-time using fluorescent substances, such as IR700, thus enabling the observation of drug distribution in tumors [14-16]. LIGHTVISION is a commercially available imaging system for indocyanine green (ICG) and has been used in basic studies of the super-enhanced permeability and retention (SUPR) effects of PIT [17]. It was reported that by using LIGHTVISION to detect the fluorescence of IR700 in real time, tumor accumulation of trastuzumab-IR700 antibody could be visualized during treatment, and the location of subcutaneous tumors in mice could be confirmed [18-20]. However, few reports have examined the relationship between the therapeutic effects of PIT and fluorescence intensity.

We hypothesized that the therapeutic effect of PIT and fluorescence intensity were correlated because IR700-conjugated antibodies aggregate and induce cell death by light irradiation and simultaneously emit fluorescence. Therefore, in this study, we performed real-time fluorescence imaging of tumors under treatment to investigate the relationship between fluorescence intensity and therapeutic efficacy. In addition, we determined the amount of light irradiation required for effective treatment based on the results.

\section{Results}

\subsection{Quality Control of Cetuximab-IR700}

Cetuximab (chimeric (mouse/human) mAb) and a control mAb (human IgG1 isotype control recombinant clone) were conjugated to IR700 to produce a new antibody drug. The schematic structure of the Cet-IR700 is shown (Figure S1). The produced antibodies, Cet-IR700 and Control mAb-IR700 (negative control), were analyzed by sodium dodecyl sulfate-polyacrylamide gel electrophoresis (SDS-PAGE). Cet-IR700 and non-conjugated cetuximab had the same molecular weight of approximately $150 \mathrm{kDa}$. The same result was observed in the Control mAb-IR700 (Figure 1A). The conjugation of cetuximab, Cet-IR700, control $\mathrm{mAb}$, and Control mAb-IR700 to A431 cells was examined by flow cytometry. Cet-IR700 and cetuximab showed a high binding ability to the EGFR protein, and the binding ability of EGFR did not change when IR700 was conjugated. In addition, Control $\mathrm{mAb}-\mathrm{IR} 700$ and control $\mathrm{mAb}$ did not bind to the EGFR protein (Figure 1B). Time-lapse fluorescence microscopy imaging was performed to examine the effect of cell membrane damage after PIT and the target-specific localization of Cet-IR700 and Control mAb-IR700. Before treatment, IR700 was mainly localized in the membrane of A431 cells. Immediately after laser exposure, A431 cells formed blisters and ruptured due to cell membrane damage. Most of these morphological changes were observed within 15 min of laser irradiation (Figure 1C). However, Control mAb-IR700 did not localize to the plasma membrane of A431 cells, and the cells did not show any changes after laser irradiation (Figure 1D). 

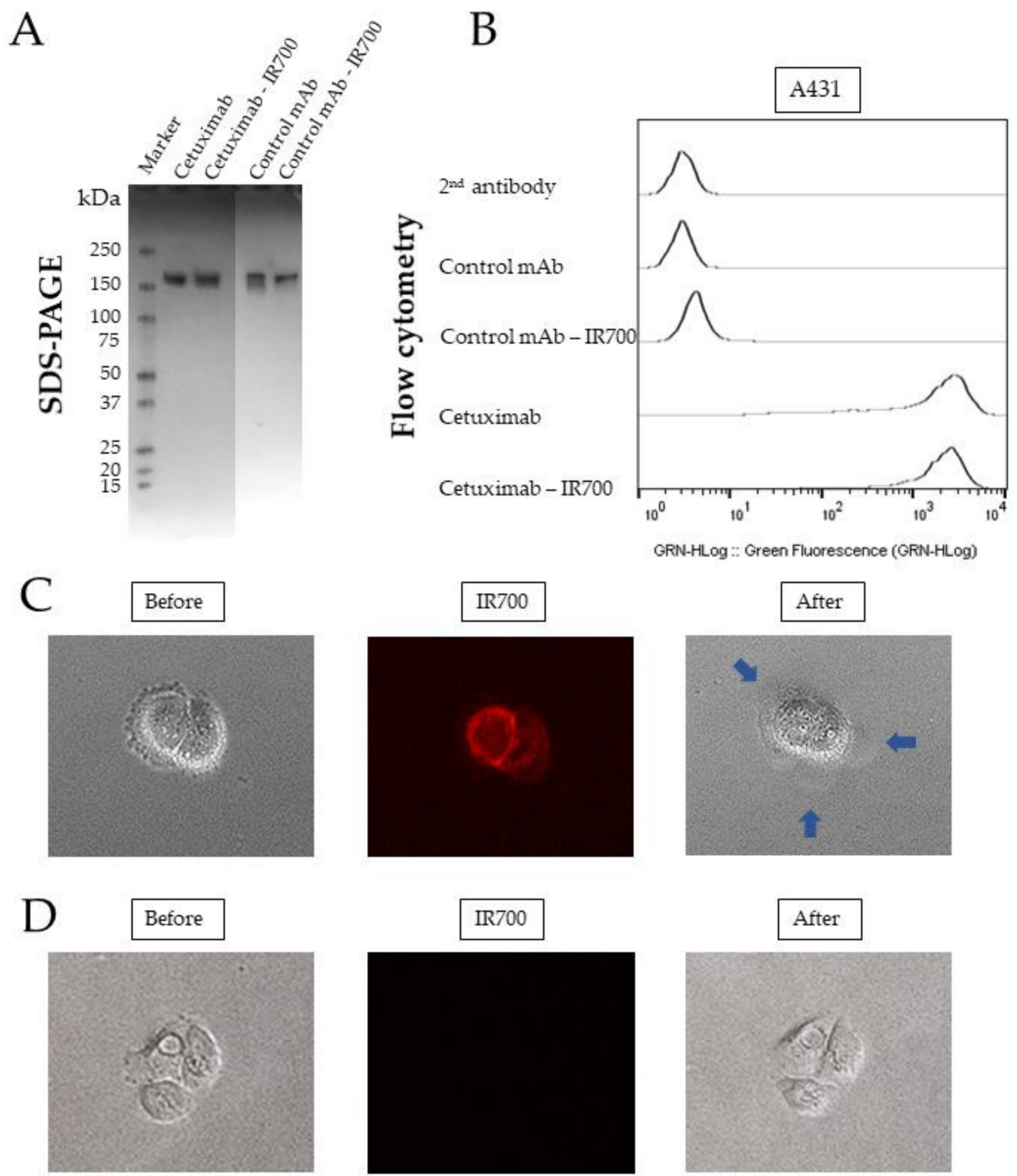

Figure 1. Quality control of Cetuximab-IR700. (A) Non-reducing SDS-PAGE analysis of Cet-IR700 and Control mAb-IR700. Marker: molecular mass marker. (B) Conjugation of various monoclonal antibodies to A431 cells. Cet-IR700 and cetuximab showed a high binding ability to EGFR protein. (C) Morphological changes of cells after photoimmunotherapy (PIT) with Cet-IR700. The EGFRspecific localization of Cet-IR700 in A431 cells was confirmed by fluorescence microscopy. Ruptured A431 cells were observed under the microscope after $20 \mathrm{~J} / \mathrm{cm}^{2}$ of laser irradiation. Blue arrows indicate cells that have ruptured and formed blisters. (D) Morphological changes of cells after PIT with Control mAb-IR700. There was no morphological change of A431 cells before and after PIT, and Control mAb-IR700 could not be observed by fluorescence microscopy. 


\subsection{In Vivo Real-Time Fluorescence Imaging during PIT}

The LIGHTVISION camera is a system for capturing and imaging the fluorescence of indocyanine green (peak fluorescence: $830 \mathrm{~nm}$ ). Because the fluorescence spectrum of Cet-IR700 extends past $800 \mathrm{~nm}$, it was assumed that the LIGHTVISION camera could detect IR700 fluorescence. Therefore, we set out to confirm whether the LIGHTVISION camera can detect tumors by fluorescence observation when a $690 \mathrm{~nm}$ laser is used to irradiate the tumor. The schema of the fluorescence imaging experiment is shown in Figure 2A. Briefly, a cylindrical catheter was punctured along the bottom or side of the tumor in the mice (not into the tumor), and a diffuser was inserted into the catheter to deliver laser radiation (Figure 2B,C), while a frontal diffuser delivered surface radiation to the tumor (Figure 2B). When the subcutaneous tumors of the mice were irradiated with $690 \mathrm{~nm}$ light $24 \mathrm{~h}$ after the administration of Cet-IR700 (100 $\mu \mathrm{g} /$ animal), the tumor accumulation of the antibody could be visualized, and the location of the tumor could be confirmed. Next, real-time fluorescence imaging was performed on the tumors bound with Cet-IR700, and the fluorescence images at irradiation levels of 0,10, 40,60, and $100 \mathrm{~J} / \mathrm{cm}$ were compared. The results showed that the fluorescence intensity of the tumor was at a maximum at the beginning of administration and gradually decreased as the laser dose increased (Figure 2D).

A

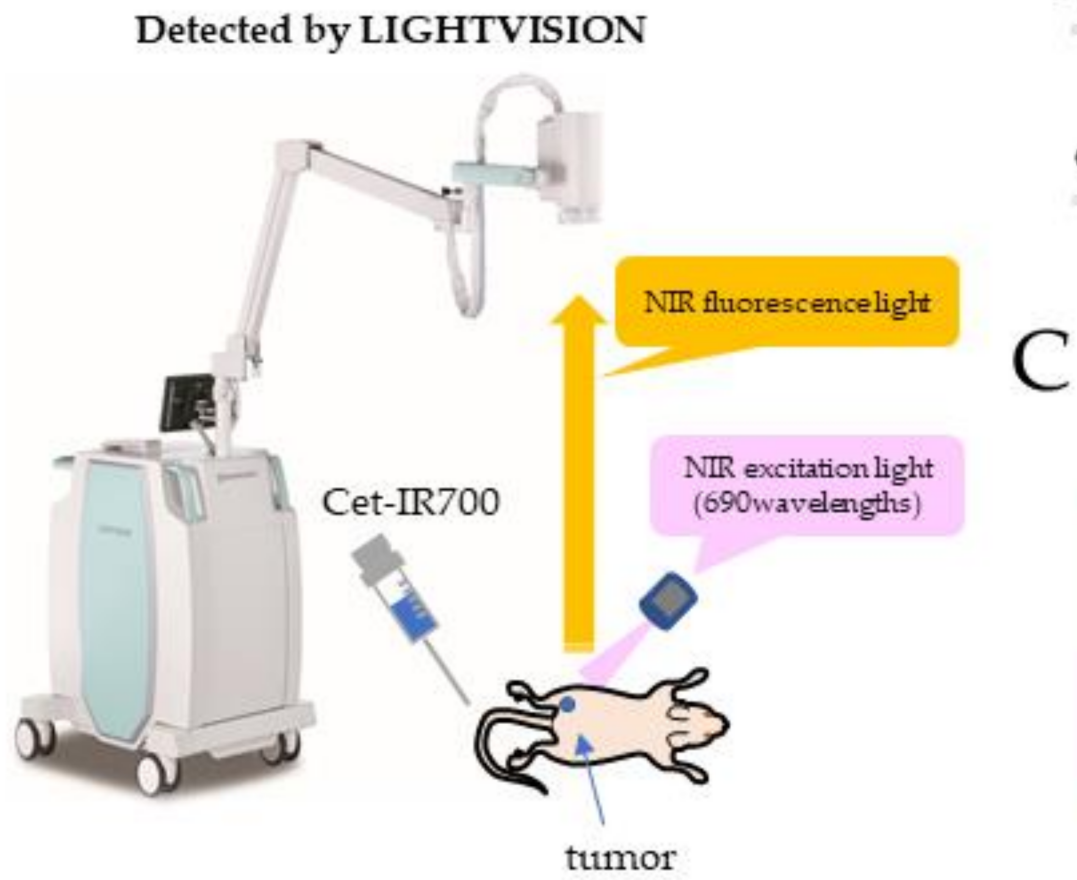

Frontal diffuser

\section{Cylindrical diffuser}
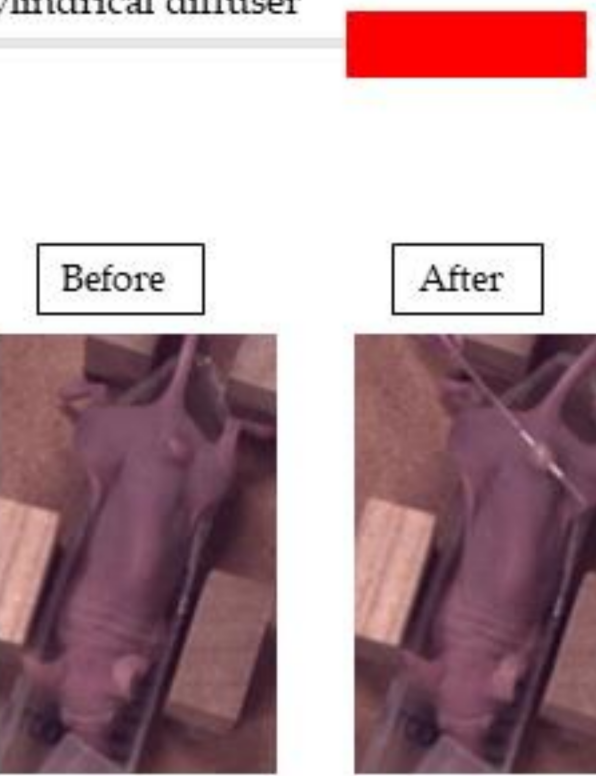

\section{After}

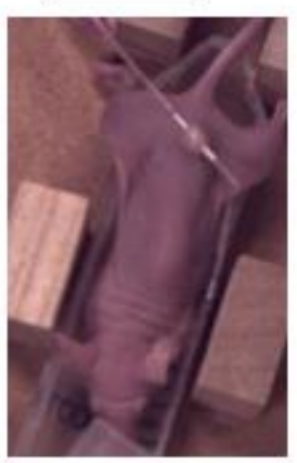

Figure 2. Cont. 

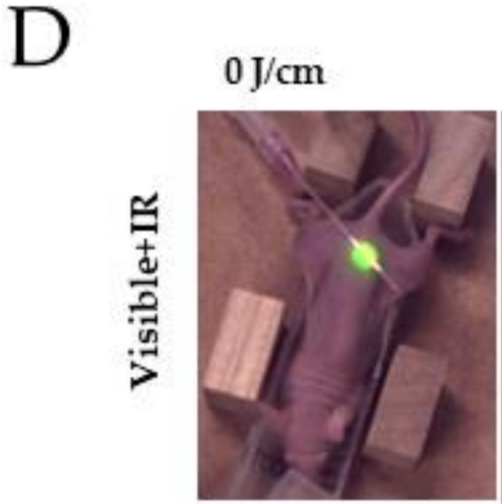
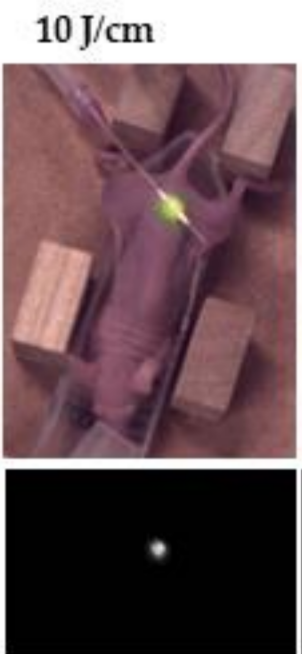

\section{$40 \mathrm{~J} / \mathrm{cm}$}

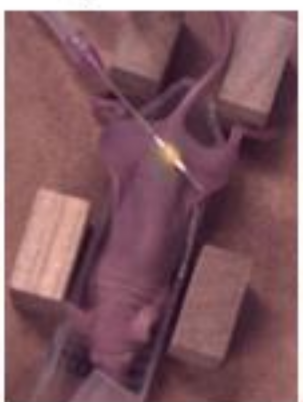

$60 \mathrm{~J} / \mathrm{cm}$
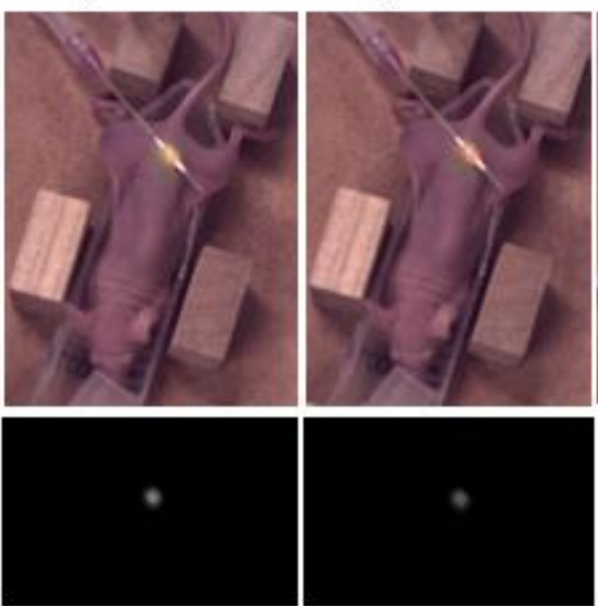

$100 \mathrm{~J} / \mathrm{cm}$
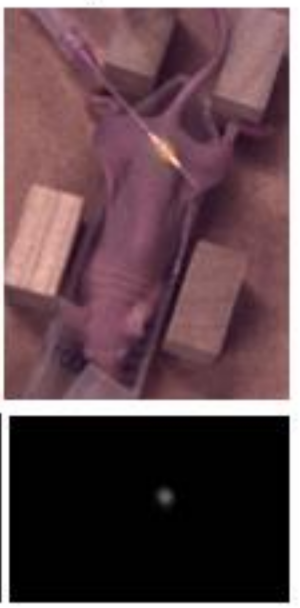

Figure 2. In vivo real-time fluorescence imaging during PIT. (A) Experimental setup for fluorescence observation using LIGHTVISION. (B) The frontal diffuser provided surface irradiation to tumors and was used for in vitro experiments and tissue evaluation. The unit of irradiation dose was $\mathrm{J} / \mathrm{cm}^{2}$. The cylindrical diffuser provided intra-tissue irradiation of the tumor and was used for in vivo imaging and antitumor efficacy studies. The unit of irradiation dose was J/cm. (C) Cylindrical catheter puncture method for subcutaneous tumors in mice. The subcutaneous tissue proximal to the tumor was punctured. (D) Mice with a tumor volume of approximately $100 \mathrm{~mm}^{3}$ were treated with $100 \mu \mathrm{g}$ of Cet-IR700. After $24 \mathrm{~h}$, A431 tumors were irradiated with light of a $690 \mathrm{~nm}$ wavelength, and fluorescence images were captured by LIGHTVISION. The fluorescence of Cet-IR700 accumulated in the tumor during laser irradiation was detected.

\subsection{Real-Time Fluorescence Imaging Analysis of IR700}

Nine mice were treated with PIT, and real-time fluorescence imaging was performed on Cet-IR700-bound tumors. The average fluorescence intensity was calculated by imaging the region of interest of the tumor during treatment, and a graph was created with the $x$-axis corresponding to the laser dosage. Fluorescence intensity analysis of IR700 showed a sharp decrease in the early stages of treatment, and the decrease slowed when the dose reached $40 \mathrm{~J} / \mathrm{cm}$ (Figure 3A). Averaging the fluorescence intensities of the nine mice, we found that as the radiation dose increased to $60-100 \mathrm{~J} / \mathrm{cm}$, the fluorescence intensity decreased to about 20\%, almost reaching a plateau (Figure 3B). Comparing the percentage of fluorescence decay at each irradiation dose, the 10 and $40 \mathrm{~J} / \mathrm{cm}$ groups showed significant differences compared to the $100 \mathrm{~J} / \mathrm{cm}$ group ( $n=9, p<0.001$ and $p=0.003$, respectively). The 100,80 , and $60 \mathrm{~J} / \mathrm{cm}$ groups showed no significant difference, and the decay rate of fluorescence intensity was almost in equilibrium (Figure 3C). 
A

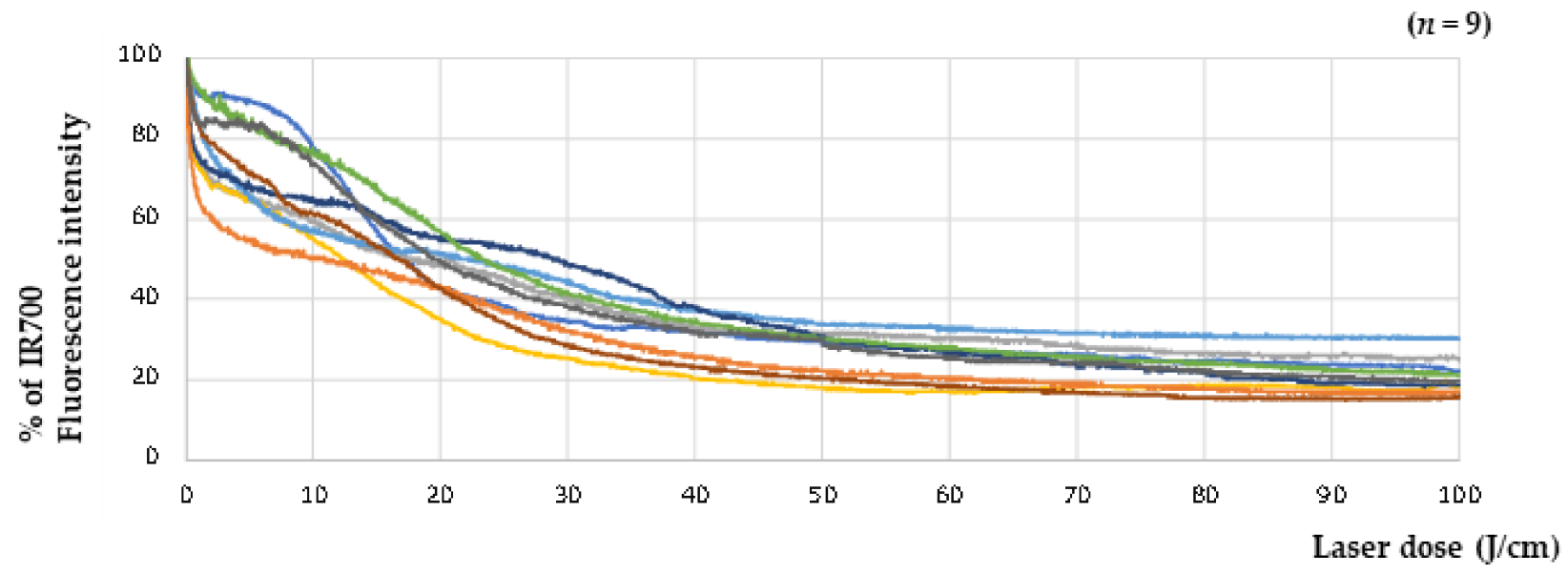

B

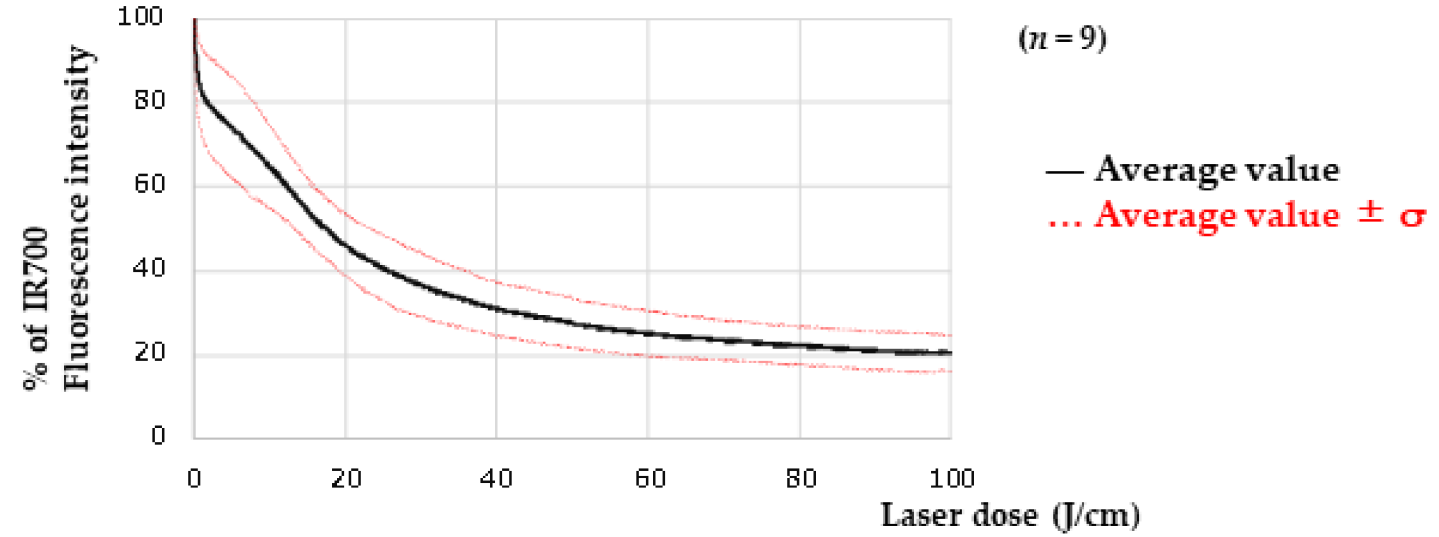

C

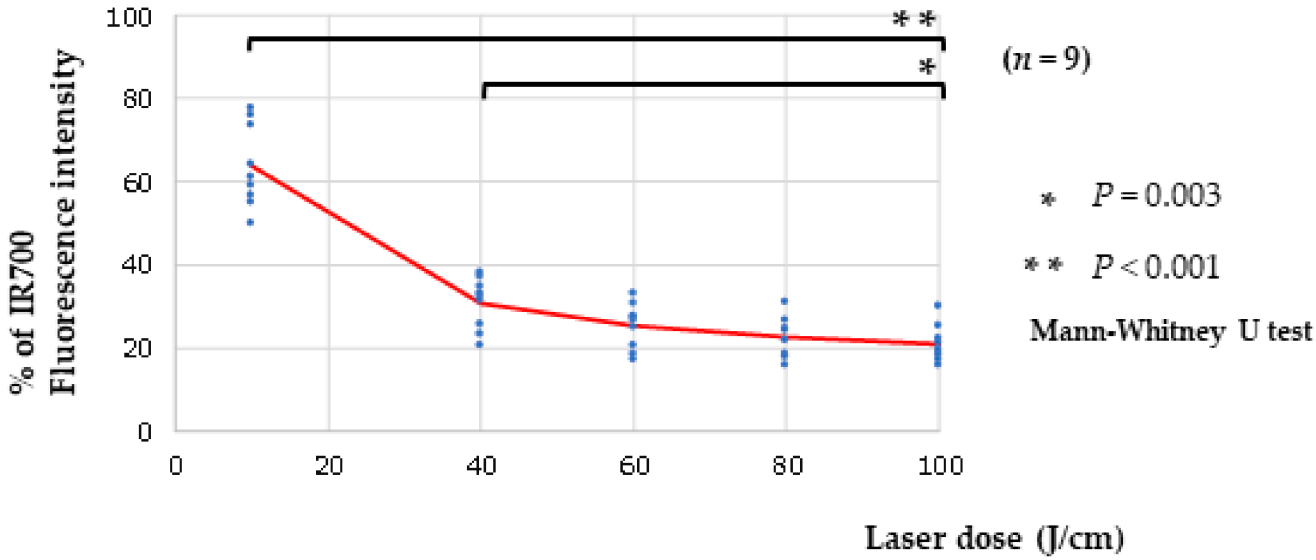

Figure 3. Real-time fluorescence imaging analysis of IR700. (A) The time fluorescence intensity curve shows the calculated average fluorescence intensity of Cet-IR700-bound tumors during PIT. (B) The mean values of the time fluorescence intensity curves and the mean $\pm \sigma$ (red dots) are shown. (C) The rate of decay of fluorescence intensity was compared between the clinical dose of $100 \mathrm{~J} / \mathrm{cm}$ and the $10,40,60$, and $80 \mathrm{~J} / \mathrm{cm}$ groups $(n=9, p<0.001, p=0.003, p=0.10$, and $p=0.51$, respectively, analyzed by the Mann-Whitney U test). 


\subsection{Relationship between Laser Dose and Antitumor Effect}

The relationship between the laser dose and the antitumor effect was evaluated. Based on the decay rate of the fluorescence intensity, the laser doses of $10 \mathrm{~J} / \mathrm{cm}$, when the fluorescence intensity rapidly declined, $40 \mathrm{~J} / \mathrm{cm}$, just before the fluorescence intensity plateaued, and $60 \mathrm{~J} / \mathrm{cm}$, when the fluorescence intensity had reached a plateau, were compared with the dose of $100 \mathrm{~J} / \mathrm{cm}$. First, we compared the initial fluorescence intensity in each group. The mean value of the fluorescence intensity was 182 , and there was no significant difference among the groups, indicating that the antibody accumulated in the tumor (Figure 4A). Next, we examined the rate of decay of the fluorescence intensity at the end of laser irradiation in each group and found a significant difference only between the $10 \mathrm{~J} / \mathrm{cm}$ and $100 \mathrm{~J} / \mathrm{cm}$ groups ( $p<0.004$, Mann-Whitney U test, Figure 4B). Using Cet-IR700, PIT was performed at each irradiation dose, and the effect was examined in mice with A431 tumors. The PIT group was irradiated with a laser $24 \mathrm{~h}$ after intravenous injection of Cet-IR700. The volume of A431 tumors was significantly reduced in the groups that received Cet-IR700 followed by laser irradiation $(10,40,60$, and $100 \mathrm{~J} / \mathrm{cm})$ compared to the no treatment group ( $p<0.001, p<0.01$; one-way analysis of variance, Tukey test). The antitumor effects did not differ significantly among the groups that received 40,60, and $100 \mathrm{~J} / \mathrm{cm}$ radiation. In addition, there was a trend of a decrease in tumor volume in the 40, 60 , and $100 \mathrm{~J} / \mathrm{cm}$ dose groups compared to the $10 \mathrm{~J} / \mathrm{cm}$ dose group (Figure 4C).

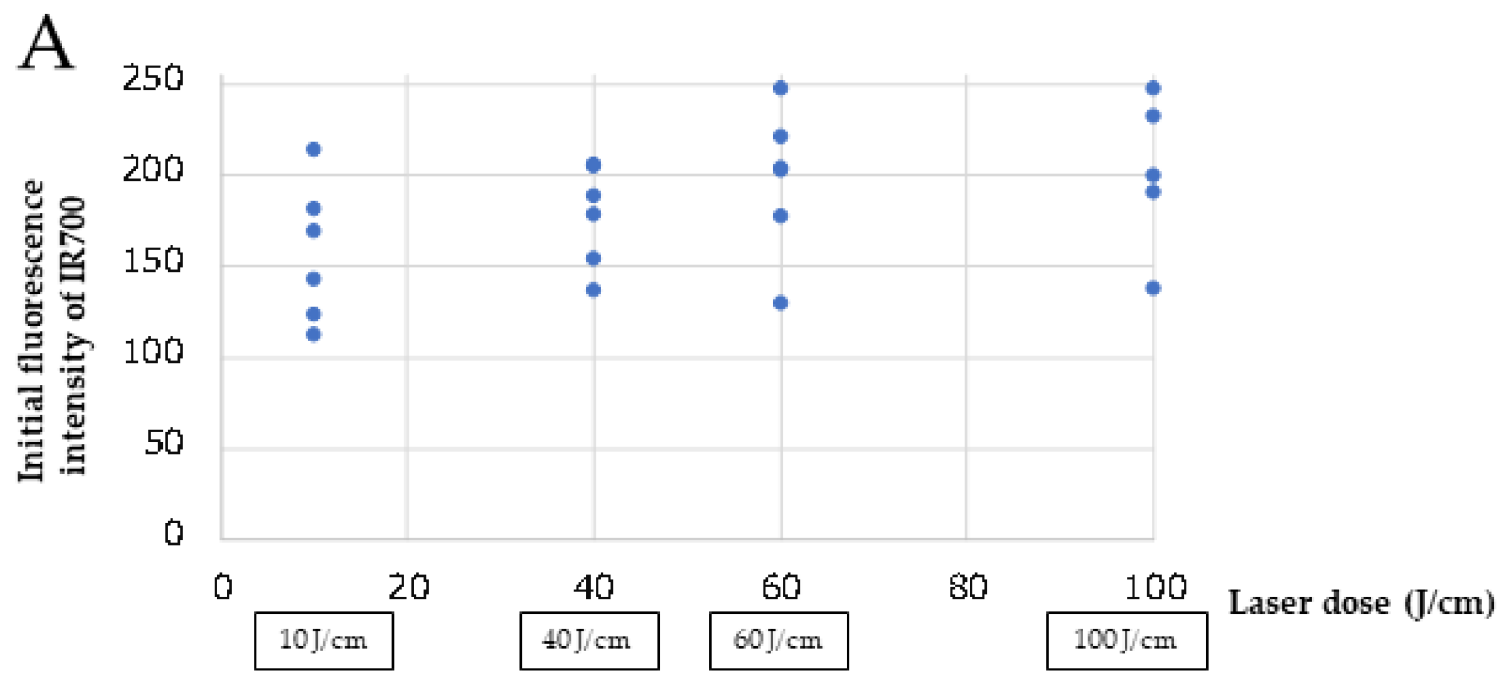

Figure 4. Cont. 

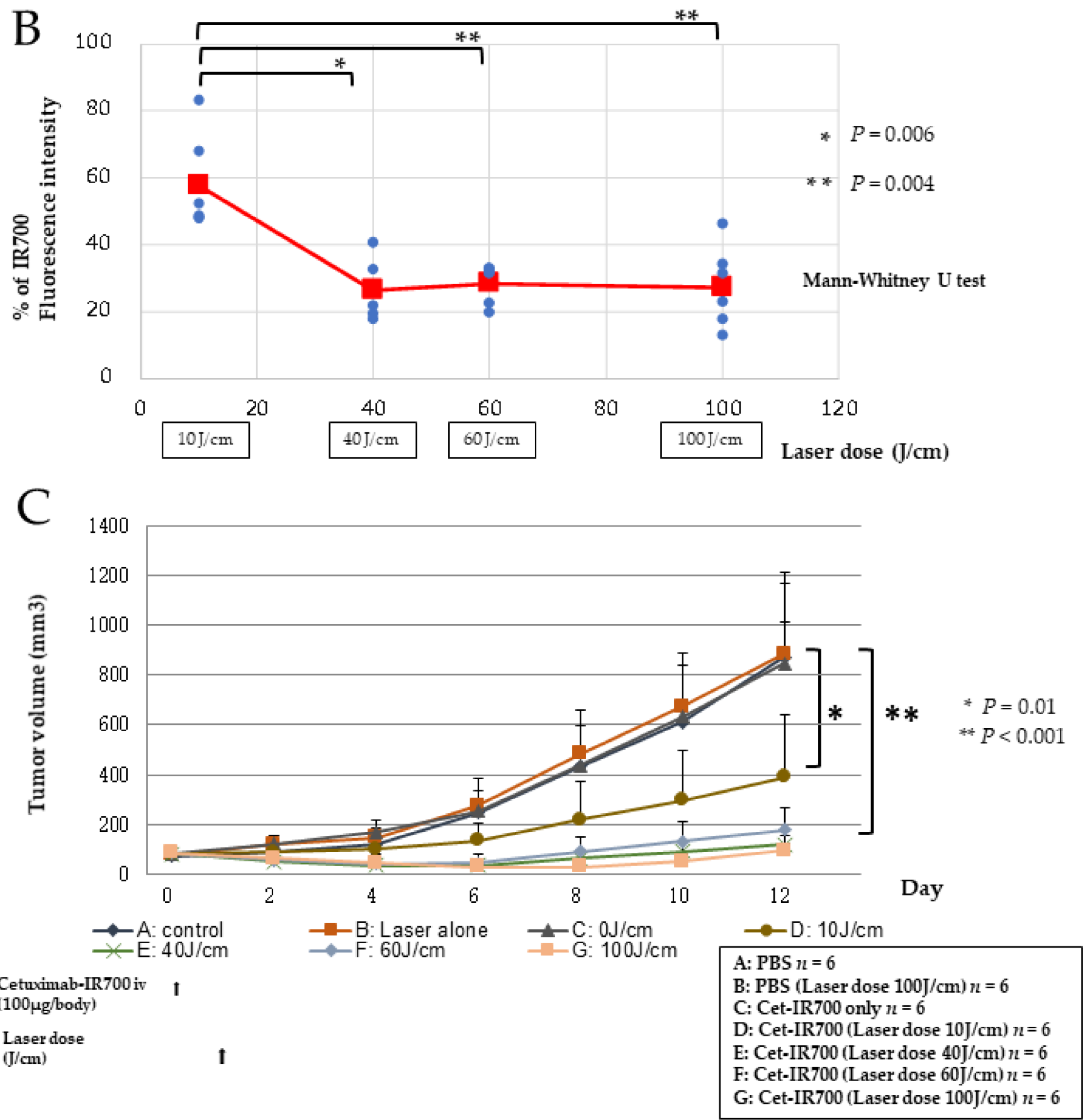

Figure 4. Relationship between laser dose and antitumor effect. (A) The fluorescence intensity at the beginning of treatment was examined in each laser dose group $(10,40,60,100 \mathrm{~J} / \mathrm{cm})$. (B) The rate of fluorescence intensity decay at the end of irradiation was examined in each laser dose group (10, 40, $60,100 \mathrm{~J} / \mathrm{cm}$ ). (C) Volumes of A431 tumors were compared among the groups treated with Cet-IR700 and irradiated with various laser doses $(10,40,60$, and $100 \mathrm{~J} / \mathrm{cm})$ and the untreated control group. Data are shown as mean values ( $n=6$ per group, 12 days after initial treatment, dots are mean values, bars are SD).

\subsection{Histological Analysis of PIT}

Histological changes $24 \mathrm{~h}$ after PIT with various laser doses were evaluated. Microscopic evaluation of the treated tumors showed scattered clusters of damaged tumor cells after PIT of $10-100 \mathrm{~J} / \mathrm{cm}^{2}$. The damaged cells had eosinophilic cell chambers and pyknotic 
nuclei. Although there was no obvious damage in the control group, living but damaged tumor cells were scattered against a background of microhemorrhage and inflammatory cell infiltration consistent with acute granule formation in the laser-irradiated groups. In the groups with a dose of $40 \mathrm{~J} / \mathrm{cm}^{2}$ or higher, there was no obvious difference in the remaining tumor cells according to the laser irradiance. However, the number of damaged tumor cells in the $10 \mathrm{~J} / \mathrm{cm}^{2}$ group was lower than that of the other radiation doses (Figure 5).
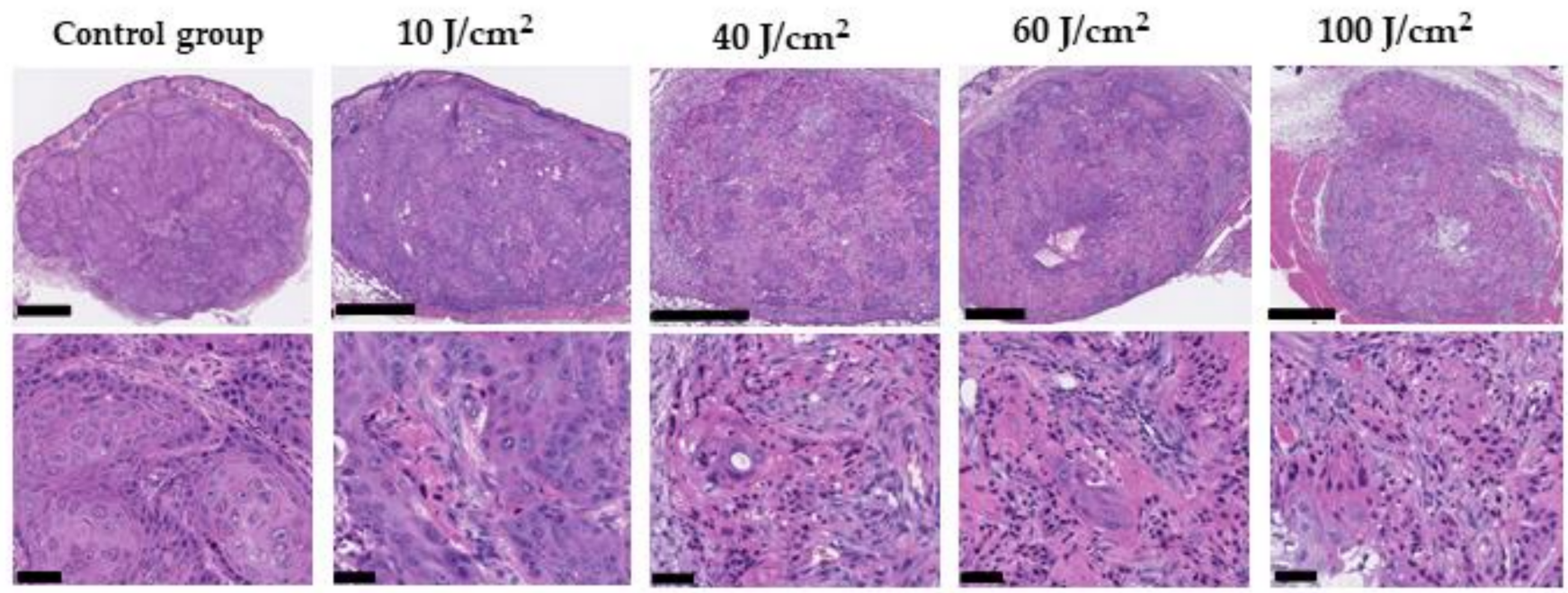

Figure 5. Histological findings $24 \mathrm{~h}$ after PIT with various laser doses. Histological specimens of A431 tumors with no treatment and PIT of $10,40,60$, and $100 \mathrm{~J} / \mathrm{cm}^{2}$ are shown. All specimens are stained with hematoxylin and eosin. Scattered clusters of damaged tumor cells are seen. (Upper black scale bar $=500 \mu \mathrm{m}$, Lower black scale bar $=50 \mu \mathrm{m}$ ).

\section{Discussion}

PIT is currently in need of an imaging method capable of confirming the location of the tumor and determining the effect of treatment. Upon light irradiation, IR700-conjugated antibodies form aggregates and cause membrane damage in cancer cells. As a result, cell death is induced. In addition, after this photochemical reaction, IR700 does not emit fluorescence due to structural changes, and the fluorescence disappears [2,3]. In our study, PIT treatment of A431 also resulted in membrane damage and loss of IR700 fluorescence after treatment (Figure 1C). And in vivo experiments showed that even if the amount of antibody accumulation in the tumor is high at the beginning of treatment, it does not necessarily mean that the treatment is highly effective (Figure S2). Not only the amount of antibody accumulation, but also the amount of light and the method of irradiation are important to enhance the therapeutic effect. Based on the above, we hypothesized that real-time fluorescence imaging of tumors under PIT treatment and measurement of fluorescence intensity could provide information on the treatment effectiveness of PIT.

In this study, we performed real-time fluorescence imaging of IR700 using commercially available LIGHTVISION, commonly used for ICG. Because the fluorescence peak of IR700 $(702 \mathrm{~nm})$ is more than $100 \mathrm{~nm}$ from the fluorescence peak of ICG $(805-845 \mathrm{~nm})$ [21], the imaging equipment for ICG was not sensitive enough for IR700, proving to be a challenge for fluorescence imaging of IR700. Nevertheless, the location of the tumor was clearly identified in this study (Figure 2D). Although the wavelengths were far from the fluorescence peak of IR700, the absence of collision with the excitation light $(690 \mathrm{~nm})$ and the low influence of autofluorescence were considered to have led to good fluorescence imaging of IR700. We also succeeded in performing real-time fluorescence imaging of the tumor during PIT therapy and found that the fluorescence gradually disappeared (Figure 2D). Analysis of IR700 during treatment showed that the fluorescence intensity decreased sharply initially, and from approximately $50 \mathrm{~J} / \mathrm{cm}$, the decay of fluorescence intensity reached near-equilibrium (Figure 3A). Therefore, it was expected that under 
conditions of rapidly changing fluorescence intensity, active cancer cell membrane damage would occur, and under equilibrium conditions of fluorescence intensity, cancer cell membrane damage would decrease. From the results shown in Figure 3A, the laser dose was determined by the rate of fluorescence decay. Then, Cet-IR700 was selectively accumulated in A431 tumors, and the antitumor effect was examined at various irradiation doses. The low dose of $10 \mathrm{~J} / \mathrm{cm}$ showed a significant antitumor effect compared to no treatment, but the therapeutic effect was insignificant compared to the dose of 40-100 J/cm, where the fluorescence intensity reached equilibrium. Moreover, there was no significant difference in the antitumor effect at doses of 40-100 J/cm. These results further confirm the relationship between the decay rate of fluorescence intensity and the antitumor effect (Figure $4 \mathrm{~B}, \mathrm{C}$ ). With respect to the irradiation dose, it was observed that dose-dependent cell death occurred when the fluorescence intensity decreased; however, when the fluorescence intensity decay reached equilibrium, the antitumor effect did not change regardless of the irradiation dose. Therefore, in addition to avoiding side effects induced by high irradiance, it has been reported that irradiance of $50 \mathrm{~J} / \mathrm{cm}^{2}$ is suitable for inducing the SUPR effect [22-24]. It is a technical application to monitor the laser dose during PIT.

This study has several limitations. First, we only examined the feasibility of this method for a mouse cell line-derived xenograft model. The use of the patient-Derived Xenograft Model would provide a more similar experiment to the human condition. Second, the average tumor size we studied was approximately $100 \mathrm{~mm}^{3}$, which does not reflect the repertoire of different tumor sizes. Fluorescence imaging may result in variable fluorescence intensity measurements depending on the tumor type and size. Because the intensity of light decreases through tissue, if the tumor is thick or large, it may not be possible to measure the fluorescence of IR700 binding antibodies that accumulate in the tumor. However, compared to previously reported fluorescence imaging studies of PIT, our study was performed under conditions of reduced light attenuation by irradiating the tumor with intra-tissue irradiation rather than surface irradiation [17-20]. Third, we used LIGHTVISION, a fluorescence imaging device that detects ICG, and obtained good results. However, similar ICG imaging devices are available $[25,26]$, but we did not study the differences between these devices.

\section{Materials and Methods}

\subsection{Cells and Cell Culture}

The A431 cell line used in this experiment was purchased from the American Type Culture Collection (Manassas, VA, USA). These cell lines were cultured in Dulbecco's Modified Eagle Medium (DMEM) (FUJIFILM Wako Pure Chemical Corporation, Osaka, Japan) supplemented with 10\% fetal bovine serum (FBS, Thermo Fisher Scientific, Waltham, MA, USA) and $1 \%$ penicillin-streptomycin-amphotericin B suspension (FUJIFILM Wako Pure Chemical Corporation, Osaka, Japan) at $37^{\circ} \mathrm{C}$ in a $5 \% \mathrm{CO}_{2}$ atmosphere.

\subsection{Synthesis of IR700-Conjugated Antibodies}

The antibody used was cetuximab (Merck Biopharma, Tokyo, Japan), a human/mouse chimeric monoclonal antibody of the IgG1 subclass that targets EGFR. Ultra-LEAFTM Purified Human IgG1 Isotype Control Recombinant (BioLegend, San Diego, CA, USA) was used as a control antibody. The photosensitive material used was IRDye 700DX NHS Ester (IR700; C74H96N12Na4O27S6Si3, molecular weight: 1954.22), purchased from LI-COR Bioscience (Lincoln, NE, USA). All reagent-grade chemicals were used in this study. The IRDye $^{\circledR}$ 700DX Protein Labeling Kit (LI-COR Bioscience, Lincoln, NE, USA) was used to bind IR700 to the antibody according to a previously established method $[27,28]$. Briefly, $1 \mathrm{mg}(6.6 \mathrm{nmol})$ of cetuximab was incubated with $53.2 \mu \mathrm{g}(27.2 \mathrm{nmol})$ of IR700. The mixture was then purified on a Zeba ${ }^{\mathrm{TM}}$ Desalting Spin Column, 7K MWCO (Thermo Fisher Scientific), to produce Cet-IR700. The control antibody was prepared in the same way to produce Con-IR700. The number of fluorophore molecules bound to each antibody molecule was determined using the following method. A spectrophotometer (NanoDrop 
One; Thermo Fisher Scientific) was used to measure the concentration of protein and IR700 by measuring the absorption at $280 \mathrm{~nm}$ and $689 \mathrm{~nm}$. On average, two IR700 molecules were bound to each antibody. Non-reducing SDS-PAGE was used to analyze the resulting conjugated antibodies.

\subsection{Flow Cytometry}

Flow cytometry was conducted as previously described $[29,30]$. Cultured cells were stained using primary antibodies (cetuximab, Cet-IR700, control antibody, Control mAbIR700) and Alexa Fluor 488-conjugated anti-rat as secondary antibodies. Stained cells were analyzed using Guava easyCyte 10HT (Merck Millipore, Billerica, MA, USA). The data were analyzed using FlowJo software (Tree Star Inc., Ashland, OR, USA).

\subsection{In Vitro Fluorescence Microscopic Images and PIT}

Fluorescence microscopy was performed using a BZ-X700 fluorescence microscope (Keyence, Osaka, Japan). The molecular target-specific localization of Cet-IR700 and Control mAb-IR700 was confirmed in A431 cells. Cells were seeded on 96-well optical-bottom plates with a polymer base (Thermo Fisher Scientific) and incubated at $37^{\circ} \mathrm{C}$ for $24-48 \mathrm{~h}$. Cet-IR700 $(10 \mu \mathrm{g} / \mathrm{mL})$ or Control mAb-IR700 $(10 \mu \mathrm{g} / \mathrm{mL})$ was added to the culture medium and incubated for $3 \mathrm{~h}$ at $37^{\circ} \mathrm{C}$. The cells were then washed with phosphate-buffered saline (PBS) and observed under a fluorescence microscope. Next, they were laser irradiated with a $690 \mathrm{~nm}$ continuous wave laser (MLL-III-690; CNI Optoelectronics Technology, Changchun, China). A power density of $30 \mathrm{~mW} / \mathrm{cm}^{2}$ was measured using an optical power meter (PM100, Thorlabs, Newton, NJ, USA). The laser dose was $20 \mathrm{~J} / \mathrm{cm}^{2}$. The cells before and after irradiation were observed under a microscope.

\subsection{Animal Model}

Six-week-old female BALB/c nu/nu mice (Charles River Japan, Yokohama, Japan) were purchased. Mice were anesthetized with isoflurane and inoculated with $3.5 \times 10^{6} \mathrm{~A} 431$ cells suspended in $100 \mu \mathrm{L}$ of PBS on the left dorsal side. Tumor volume was calculated using the following equation: TV $=\left(L \times W^{2}\right) / 2$, where $L$ and $W$ are the length and width of the tumor under the skin, respectively [31]. Tumor volume and weight were measured every two days, and mice that reached $1000 \mathrm{~mm}^{3}$ or lost more than $20 \%$ of their body weight were euthanized due to humane treatment. Animal experiments were approved by the Animal Experiment Committee of the National Cancer Center Japan. All animal experiments were performed in accordance with the Guidelines for the Care and Use of Laboratory Animals established by the committee. These guidelines meet the ethical standards set forth by law and comply with the Japanese Guidelines for the Use of Laboratory Animals.

\subsection{In Vivo Real-Time Fluorescence Imaging}

To perform fluorescence imaging during PIT, LIGHTVISION (Shimadzu Corporation, Kyoto, Japan) was used at a collection wavelength of $820 \mathrm{~nm}$ or higher, and the excitation light was a $690 \mathrm{~nm}$ laser $(150 \mathrm{~mW} / \mathrm{cm})$ from a laser irradiation device (MLL-III-690) used in PIT therapy. A cylindrical diffuser, which emits light from the laser device to the tumor, was placed alongside the tumor (no diffuser was placed inside the tumor), and intra-tissue irradiation was performed. The LIGHTVISION camera head was placed approximately $50 \mathrm{~cm}$ from the observation target. Mice with A431 tumors of approximately $100 \mathrm{~mm}^{3}$ were injected with $100 \mu \mathrm{g}$ of Cet-IR700, and $24 \mathrm{~h}$ later, PIT treatment was performed to obtain fluorescence images and tumor fluorescence intensity during treatment. The exposure time, camera sensitivity, binning, and other conditions were observed under the same settings, and fluorescence imaging was performed continuously. The obtained fluorescence images were analyzed by Fiji software [32]. After setting the region of interest for each tumor region, the pixel values were measured, and a graph was created with the x-axis corresponding to the light intensity. All pixel values were normalized to a maximum intensity of $100 \%$. 


\subsection{In Vivo PIT with Cet-IR700}

In vivo experiments were performed using mice implanted with A431 cells. Mice with A431 tumor xenografts and a tumor volume of approximately $100 \mathrm{~mm}^{3}$ were selected and randomly divided into seven groups ( $n=6$ mice per group). The seven groups were as follows: (a) No treatment (intravenous injection of PBS without laser irradiation). (b) Laser irradiation (100 J/ cm $24 \mathrm{~h}$ after intravenous injection of PBS. (c) Intravenous injection of $100 \mu \mathrm{g}$ of Cet-IR700 without laser irradiation. (d) Laser irradiation $(10 \mathrm{~J} / \mathrm{cm}) 24 \mathrm{~h}$ after intravenous injection of $100 \mu \mathrm{g}$ Cet-IR700. (e) Laser irradiation $(40 \mathrm{~J} / \mathrm{cm}) 24 \mathrm{~h}$ after intravenous injection of $100 \mu \mathrm{g}$ Cet-IR700. (f) Laser irradiation $(60 \mathrm{~J} / \mathrm{cm}) 24 \mathrm{~h}$ after intravenous injection of $100 \mu \mathrm{g}$ Cet-IR700. (g) Laser irradiation $(100 \mathrm{~J} / \mathrm{cm}) 24 \mathrm{~h}$ after intravenous injection of $100 \mu \mathrm{g}$ Cet-IR700. Laser irradiation was performed under isoflurane anesthesia, and a $690 \mathrm{~nm}$ continuous wave laser was used at a power density of $150 \mathrm{~mW} / \mathrm{cm}$. In addition, a cylindrical diffuser was placed near the tumor (the diffuser was not inserted inside the tumor), and intra-tissue irradiation was performed. After PIT treatment, the tumor volume was measured once every two days until the tumor volume reached $1000 \mathrm{~mm}^{3}$.

\subsection{Histological Analysis}

A series of histological changes $24 \mathrm{~h}$ after PIT with various laser doses were evaluated. Mice with A431 tumors were euthanized after $24 \mathrm{~h}$ of irradiation at 40,60 , and $100 \mathrm{~J} / \mathrm{cm}^{2}$. Laser irradiation was performed using a frontal diffuser to avoid any physical effects of the diffuser on the tumors, and a $690 \mathrm{~nm}\left(150 \mathrm{~mW} / \mathrm{cm}^{2}\right)$ laser was used. The tumors were then excised, fixed in $10 \%$ formalin, and embedded in paraffin. Serial sections $(4 \mu \mathrm{m})$ were fixed on glass slides and stained with hematoxylin and eosin. Pathological images were analyzed using Nano Zoomer 2.0HT (Hamamatsu Photonics, Hamamatsu, Japan) and the image viewing software NDP.view2 (Hamamatsu Photonics).

\subsection{Statistical Analysis}

Statistical analyses were performed using EZR, a modified version of R commander designed to add statistical functions frequently used in biostatistics [33]. For multiple comparisons with the control group, one-way analysis of variance. The Mann-Whitney U test was used to compare the decay rate of fluorescence intensity with the amount of laser irradiation. Statistical significance was set at $p<0.05$.

\section{Conclusions}

In conclusion, we have successfully visualized target tumors during PIT using CetIR700 with LIGHTVISION. Analysis of the fluorescence intensity of tumors during PIT showed a relationship between the decay rate of the fluorescence intensity and the antitumor effect. As for the laser dose, it was suggested that dose-dependent cell death occurs when the fluorescence intensity decays; however, when the fluorescence intensity decay reaches equilibrium, the therapeutic effect may remain the same regardless of the dose.

Supplementary Materials: The following supporting information can be downloaded at: https: / / www.mdpi.com/article/10.3390/ph15020223/s1, Figure S1: Schematic structures of Cet-IR700, Figure S2: Relationship between Initial fluorescence intensity of IR700 and antitumor effect.

Author Contributions: Conceptualization: K.T., Y.K., A.I., and T.Y. (Tomonori Yano); Methodology: K.T., Y.K., T.A., M.Y. and T.Y. (Tomonori Yano); Analysis, investigation, and data curation: K.T., Y.K., T.A., K.M., T.Y. (Toru Yamaguchi), A.I., S.S., M.Y. and T.Y. (Tomonori Yano); Writing-original draft preparation: K.T.; Writing-review and editing: K.T., Y.K. and T.Y. (Tomonori Yano); Supervision: Y.K. and T.Y. (Tomonori Yano); Funding Acquisition: T.Y. (Tomonori Yano). All authors have read and agreed to the published version of the manuscript.

Funding: This work was financially supported in part by a joint research grant from the National Cancer Center and Shimadzu Corporation (C2021-002). 
Institutional Review Board Statement: The study was conducted according to the ethical standards set forth by law and comply with the Japanese Guidelines for the Use of Laboratory Animals, and approved by the Animal Experiment Committee of the National Cancer Center Japan (protocol code K21-010 and approval date on 4 March 2021).

Informed Consent Statement: Not applicable.

Data Availability Statement: The data that support the findings of this study are available from the corresponding author, [Tomonori Yano], upon reasonable request.

Acknowledgments: The authors thank Yasunaga's laboratory members for their helpful discussion and Mamada for her secretarial support.

Conflicts of Interest: T.Y. (Tomonori Yano) has a joint research agreement with Shimadzu Corporation. K.M., T.Y. (Toru Yamaguchi), and A.I. are employees of Shimadzu Corporation. The other authors declare no conflicts of interest.

\section{References}

1. Mitsunaga, M.; Ogawa, M.; Kosaka, N.; Rosenblum, L.T.; Choyke, P.L.; Kobayashi, H. Cancer cell-selective in vivo near infrared photoimmunotherapy targeting specific membrane molecules. Nat. Med. 2011, 17, 1685-1691. [CrossRef] [PubMed]

2. Sato, K.; Ando, K.; Okuyama, S.; Moriguchi, S.; Ogura, T.; Totoki, S.; Hanaoka, H.; Nagaya, T.; Kokawa, R.; Takakura, H.; et al. Photoinduced Ligand Release from a Silicon Phthalocyanine Dye Conjugated with Monoclonal Antibodies: A Mechanism of Cancer Cell Cytotoxicity after Near-Infrared Photoimmunotherapy. ACS Cent. Sci. 2018, 4, 1559-1569. [CrossRef] [PubMed]

3. Nakajima, K.; Takakura, H.; Shimizu, Y.; Ogawa, M. Changes in plasma membrane damage inducing cell death after treatment with near-infrared photoimmunotherapy. Cancer Sci. 2018, 109, 2889-2896. [CrossRef] [PubMed]

4. Shimoyama, K.; Kagawa, S.; Ishida, M.; Watanabe, S.; Noma, K.; Takehara, K.; Tazawa, H.; Hashimoto, Y.; Tanabe, S.; Matsuoka, J.; et al. Viral transduction of the HER2-extracellular domain expands trastuzumab-based photoimmunotherapy for HER2-negative breast cancer cells. Breast Cancer Res. Treat. 2015, 149, 597-605. [CrossRef] [PubMed]

5. Watanabe, R.; Hanaoka, H.; Sato, K.; Nagaya, T.; Harada, T.; Mitsunaga, M.; Kim, I.; Paik, C.H.; Wu, A.M.; Choyke, P.L.; et al. Photoimmunotherapy targeting prostate-specific membrane antigen: Are antibody fragments as effective as antibodies? J. Nucl. Med. 2015, 56, 140-144. [CrossRef]

6. Antonioli, L.; Pacher, P.; Vizi, E.S.; Haskó, G. CD39 and CD73 in immunity and inflammation. Trends Mol. Med. 2013, 19, 355-367. [CrossRef]

7. Sato, K.; Sato, N.; Xu, B.; Nakamura, Y.; Nagaya, T.; Choyke, P.L.; Hasegawa, Y.; Kobayashi, H. Spatially selective depletion of tumor-associated regulatory T cells with near-infrared photoimmunotherapy. Sci. Transl. Med. 2016, 8, 352ra110. [CrossRef]

8. Shirasu, N.; Yamada, H.; Shibaguchi, H.; Kuroki, M.; Kuroki, M. Potent and specific antitumor effect of CEA-targeted photoimmunotherapy. Int. J. Cancer 2014, 135, 2697-2710. [CrossRef]

9. Hanaoka, H.; Nakajima, T.; Sato, K.; Watanabe, R.; Phung, Y.; Gao, W.; Harada, T.; Kim, I.; Paik, C.H.; Choyke, P.L.; et al. Photoimmunotherapy of hepatocellular carcinoma-targeting Glypican-3 combined with nanosized albumin-bound paclitaxel. Nanomedicine 2015, 10, 1139-1147. [CrossRef]

10. Nagaya, T.; Nakamura, Y.; Sato, K.; Harada, T.; Choyke, P.L.; Kobayashi, H. Near infrared photoimmunotherapy of B-cell lymphoma. Mol. Oncol. 2016, 10, 1404-1414. [CrossRef]

11. Nagaya, T.; Nakamura, Y.; Sato, K.; Harada, T.; Choyke, P.L.; Hodge, J.W.; Schlom, J.; Kobayashi, H. Near infrared photoimmunotherapy with avelumab, an anti-programmed death-ligand 1 (PD-L1) antibody. Oncotarget 2017, 8, 8807-8817. [CrossRef] [PubMed]

12. Sato, K.; Choyke, P.L.; Kobayashi, H. Photoimmunotherapy of gastric cancer peritoneal carcinomatosis in a mouse model. PLoS ONE 2014, 9, e113276. [CrossRef] [PubMed]

13. Tahara, M.; Okano, S.; Enokida, T.; Ueda, Y.; Fujisawa, T.; Shinozaki, T.; Tomioka, T.; Okano, W.; Biel, M.; Ishida, K.; et al. A phase I, single-center, open-label study of RM-1929 photoimmunotherapy in Japanese patients with recurrent head and neck squamous cell carcinoma. Int. J. Clin. Oncol. 2021, 26, 1812-1821. [CrossRef] [PubMed]

14. Thankarajan, E.; Jadhav, S.; Luboshits, G.; Gellerman, G.; Patsenker, L. Quantification of Drug Release Degree In Vivo Using Antibody-Guided, Dual-NIR-Dye Ratiometric System. Anal Chem. 2021, 93, 8265-8272. [CrossRef]

15. Sato, K.; Watanabe, R.; Hanaoka, H.; Harada, T.; Nakajima, T.; Kim, I.; Paik, C.; Choyke, P.; Kobayashi, H. Photoimmunotherapy: Comparative effectiveness of two monoclonal antibodies targeting the epidermal growth factor receptor. Mol. Oncol. 2014, 8 , 620-632. [CrossRef]

16. Nagaya, T.; Sato, K.; Harada, T.; Nakamura, Y.; Choyke, P.; Kobayashi, H. Near Infrared Photoimmunotherapy Targeting EGFR Positive Triple Negative Breast Cancer: Optimizing the Conjugate-Light Regimen. PLoS ONE 2015, 10, e0136829. [CrossRef]

17. Rosenberg, A.; Fujimura, D.; Okada, R.; Furusawa, A.; Inagaki, F.; Wakiyama, H.; Kato, T.; Choyke, P.L.; Kobayashi, H. Real-Time Fluorescence Imaging Using Indocyanine Green to Assess Therapeutic Effects of Near-Infrared Photoimmunotherapy in Tumor Model Mice. Mol. Imaging 2020, 19, 1536012120934965. [CrossRef] 
18. Inagaki, F.F.; Fujimura, D.; Furusawa, A.; Okada, R.; Wakiyama, H.; Kato, T.; Choyke, P.L.; Kobayashi, H. Diagnostic imaging in near-infrared photoimmunotherapy using a commercially available camera for indocyanine green. Cancer Sci. 2021, 112, 1326-1330. [CrossRef]

19. Inagaki, F.F.; Fujimura, D.; Furusawa, A.; Okada, R.; Wakiyama, H.; Kato, T.; Choyke, P.L.; Kobayashi, H. Fluorescence Imaging of Tumor-Accumulating Antibody-IR700 Conjugates Prior to Near-Infrared Photoimmunotherapy (NIR-PIT) Using a Commercially Available Camera Designed for Indocyanine Green. Mol. Pharm. 2021, 18, 1238-1246. [CrossRef]

20. Okuyama, S.; Fujimura, D.; Inagaki, F.; Okada, R.; Maruoka, Y.; Wakiyama, H.; Kato, T.; Furusawa, A.; Choyke, P.L.; Kobayashi, H. Real-time IR700 Fluorescence Imaging During Near-infrared Photoimmunotherapy Using a Clinically approved Camera for Indocyanine Green. Cancer Diagn. Progn. 2021, 1, 29-34. [CrossRef]

21. Panzardi, G.; Donati, M.C.; Longobardi, G.; Pogi, P. Choroidal angiography with indocyanine green dye: Absorption and fluorescence techniques. Eur. J. Ophthalmol. 1992, 2, 83-85. [CrossRef] [PubMed]

22. Sano, K.; Nakajima, T.; Choyke, P.L.; Kobayashi, H. Markedly enhanced permeability and retention effects induced by photoimmunotherapy of tumors. ACS Nano 2013, 7, 717-724. [CrossRef] [PubMed]

23. Tang, Q.; Nagaya, T.; Liu, Y.; Horng, H.; Lin, J.; Sato, K.; Kobayashi, H.; Chen, Y. 3D mesoscopic fluorescence tomography for imaging micro-distribution of antibody-photon absorber conjugates during near infrared photoimmunotherapy in vivo. J. Control Release 2018, 279, 171-180. [CrossRef] [PubMed]

24. Zhang, X.; Nakajima, T.; Mizoi, K.; Tsushima, Y.; Ogihara, T. Imaging modalities for monitoring acute therapeutic effects after near-infrared photoimmunotherapy in vivo. J. Biophotonics 2021, 15, e202100266. [CrossRef] [PubMed]

25. Alander, J.T.; Kaartinen, I.; Laakso, A.; Pätilä, T.; Spillmann, T.; Tuchin, V.V.; Venermo, M.; Välisuo, P. A review of indocyanine green fluorescent imaging in surgery. Int. J. Biomed. Imaging 2012, 2012, 940585. [CrossRef]

26. Zhu, B.; Sevick-Muraca, E.M. A review of performance of near-infrared fluorescence imaging devices used in clinical studies. $\mathrm{Br}$. J. Radiol. 2015, 88, 20140547. [CrossRef]

27. Fujimoto, S.; Muguruma, N.; Okamoto, K.; Kurihara, T.; Sato, Y.; Miyamoto, Y.; Kitamura, S.; Miyamoto, H.; Taguchi, T.; Tsuneyama, K.; et al. A Novel Theranostic Combination of Near-infrared Fluorescence Imaging and Laser Irradiation Targeting c-KIT for Gastrointestinal Stromal Tumors. Theranostics 2018, 8, 2313-2328. [CrossRef]

28. Nishimura, T.; Mitsunaga, M.; Ito, K.; Kobayashi, H.; Saruta, M. Cancer neovasculature-targeted near-infrared photoimmunotherapy (NIR-PIT) for gastric cancer: Different mechanisms of phototoxicity compared to cell membrane-targeted NIR-PIT. Gastric Cancer 2020, 23, 82-94. [CrossRef]

29. Koganemaru, S.; Kuboki, Y.; Koga, Y.; Kojima, T.; Yamauchi, M.; Maeda, N.; Kagari, T.; Hirotani, K.; Yasunaga, M.; Matsumura, Y.; et al. U3-1402, a Novel HER3-Targeting Antibody-Drug Conjugate, for the Treatment of Colorectal Cancer. Mol. Cancer Ther. 2019, 18, 2043-2050. [CrossRef]

30. Yasunaga, M.; Saijou, S.; Hanaoka, S.; Anzai, T.; Tsumura, R.; Matsumura, Y. Significant antitumor effect of an antibody against TMEM180, a new colorectal cancer-specific molecule. Cancer Sci. 2019, 110, 761-770. [CrossRef]

31. Takashima, H.; Koga, Y.; Tsumura, R.; Yasunaga, M.; Tsuchiya, M.; Inoue, T.; Negishi, E.; Harada, M.; Yoshida, S.; Matsumura, Y. Reinforcement of antitumor effect of micelles containing anticancer drugs by binding of an anti-tissue factor antibody without direct cytocidal effects. J. Control Release 2020, 323, 138-150. [CrossRef] [PubMed]

32. Fiji, Image J. Available online: https: / /imagej.net/Fiji (accessed on 13 February 2022).

33. Kanda, Y. Investigation of the freely available easy-to-use software 'EZR' for medical statistics. Bone Marrow Transpl. 2013, 48, 452-458. [CrossRef] [PubMed] 